\title{
The new impact factor in 2011 of EAPCN
}

\author{
Peter Falkai · Hans-Jürgen Möller
}

Published online: 7 August 2012

(C) Springer-Verlag 2012

Just now, ISI has released the new impact factor for 2011. Actually, European Archives of Psychiatry and Clinical Neuroscience (EAPCN) has an impact factor of 3.494 and thus experienced a slight decline from 3.637 in 2010. Nevertheless, the journal was able maintaining its position in the areas clinical neurology (rank 40 of 191 journals) and psychiatry (rank 33 of 129 journals). The minor fluctuation nevertheless urges us increasing our efforts to publish high-quality manuscripts focusing on clinical and basic scientific issues. We therefore will focus on outstanding clinical studies, entailing prospective data and large sample sizes as well as effects of modern therapeutic strategies. A second key aspect will be manuscripts dealing with neurobiological insights into psychiatric diseases and contributing to unravel pathophysiological aspects. Examples for well-cited papers in EAPCN in 2009 comprise areas of stigmatization by psychiatric treatment and helpseeking intentions in depression [1], human studies on cannabis and psychosis [2] and a valuable shotgun proteomic analysis on the prefrontal cortex of schizophrenia patients [3]. Due to the short time period, manuscripts published in 2010 of course could not yet reach those citation rates. But according to a preliminary ranking, studies on the metabolic syndrome in depressed inpatients [4], effects of antipsychotics on survival of stem cells in the hippocampus [5] and neuropsychological functioning in first-episode psychosis [6] are leading in the field. We specifically thank our reviewers and editors for their

P. Falkai $(\square) \cdot$ H.-J. Möller

Department of Psychiatry and Psychotherapy,

Ludwig-Maximilians-University Munich,

Nußbaumstr. 7, 80336 Munich, Germany

e-mail: pfalkai@gwdg.de tremendous work and are looking forward to them further contributing to improve the quality of our journal in 2012.

This issue opens with Bandelow et al. [7] investigating risk factors for PTSD among German soldiers, since according to army records in 2006 and 2007 PTSD affected $0.4-0.7 \%$ of all soldiers in missions abroad. Respectively assessing all soldiers admitted to a German military hospital with PTSD $(n=117)$ during this period via questionnaires and structured interviews, the authors recommend careful psychiatric screening prior recruitment to identify persons at risk for PTSD.

Covering the past 3 decades, Pompili et al. [8] reviewed literature on the relationship between suicide and substance abuse behaviors among adolescents in respect of epidemiology, comorbidity and preventive programs. Finding a high correlation between substance abuse and suicide, they suggest applying targeted suicide prevention programs. Likewise focusing on suicide, Uhl et al. [9] measured serotonergic activity in major depression after attempted suicide or with suicidal plans via loudness dependence of auditory evoked potentials (LDAEP) at different points in time. LDAEP step by step significantly increased, suggesting reduced serotonergic activity shortly after suicide attempt. Affective symptoms such as depression, apathy, and cognitive dysfunction negatively influence the quality of life (QOL) in mentally and physically ill patients, but relationships among these symptoms and QOL in healthy persons are vague. Thus, Sawa et al. [10] explored underlying cortical mechanisms via standardized questionnaires and 24-channel near-infrared spectroscopy. Results suggest that these instruments objectively may help identifying affected individuals. After, in the 1990s, many studies had investigated effects of light therapy on seasonal affective disorder, Fischer et al. [11] conducted a survey on the development of its usage in European hospitals since then. 
The authors found considerably higher application rates compared with the last survey but claim further efforts on its propagation.

Retz et al. [12] hypothesized disturbed neuronal inhibition to underlie cortical impairment in ADHD and found confirmation after measuring late auditory evoked potentials in adult ADHD patients and controls. Eich et al. [13] again evaluated psychometric performance in adult ADHD by means of a new scale basing on the commonly used Symptom Checklist 90 Revised (SCL-90-R). Preliminary results bespeak this scale to be useful in retrospectively or prospectively rating adult ADHD in case specific ADHD assessment is missing.

Addressing Alzheimer's disease (AD), Guo et al. [14] investigated SORL1 genetic variants and cerebrospinal fluid biomarkers as major risk factors for the disorder in 105 German MCI and AD patients and found hints for the functional role of SORL1 in AD.

Finally, Linden and Muschalla [15] discuss pros and cons of standardized clinical interviews in diagnosing mental disorders, since ICD and DSM mainly emphasize disease algorithms, whereas structured diagnostic interviews are supposed to unravel the underlying criteria. Interviews of patients accompanied by a thorough clinical examination by a psychiatric expert revealed standardized diagnostic interviews to fail in terms of specific differential diagnoses and only catching unspecific syndromes. The authors recommend the inclusion of a glossary of psychopathological terms and guidelines in the ICD and DSM revisions to improve training of clinicians.

\section{References}

1. Schomerus G, Matschinger H, Angermeyer MC (2009) The stigma of psychiatric treatment and help-seeking intentions for depression. Eur Arch Psychiatry Clin Neurosci 259(5):298-306. doi:10.1007/s00406-009-0870-y

2. D'Souza DC, Sewell RA, Ranganathan M (2009) Cannabis and psychosis/schizophrenia: human studies. Eur Arch Psychiatry Clin Neurosci 259(7):413-431. doi:10.1007/s00406-009-0024-2

3. Martins-de-Souza D, Gattaz WF, Schmitt A, Rewerts C, Maccarrone G et al (2009) Prefrontal cortex shotgun proteome analysis reveals altered calcium homeostasis and immune system imbalance in schizophrenia. Eur Arch Psychiatry Clin Neurosci 259(3):151-163. doi:10.1007/s00406-008-0847-2

4. Richter N, Juckel G, Assion H-J (2010) Metabolic syndrome: a follow-up study of acute depressive inpatients. Eur Arch Psychiatry Clin Neurosci 260(1):41-49. doi:10.1007/s00406-0090013-5

5. Keilhoff G, Grecksch G, Bernstein H-G, Roskoden T, Becker A (2010) Risperidone and haloperidol promote survival of stem cells in the rat hippocampus. Eur Arch Psychiatry Clin Neurosci 260(2):151-162. doi:10.1007/s00406-009-0033-1

6. Zabala A, Rapado M, Arango C, Robles O, de la Serna E et al (2010) Neuropsychological functioning in early-onset first-episode psychosis: comparison of diagnostic subgroups. Eur Arch Psychiatry Clin Neurosci 260(3):225-233. doi:10.1007/s00406009-0046-9

7. Bandelow B, Koch M, Zimmermann P, Biesold K-H, Wedekind D et al (2012) Posttraumatic stress disorder (PTSD) in the German Armed Forces: a retrospective study in inpatients of a German Army hospital. Eur Arch Psychiatry Clin Neurosci. doi: 10.1007/s00406-012-0289-8

8. Pompili M, Serafini G, Innamorati M, Biondi M, Siracusano A et al (2012) Substance abuse and suicide risk among adolescents. Eur Arch Psychiatry Clin Neurosci. doi:10.1007/s00406-0120292-0

9. Uhl I, Illes F, Graßnickel V, Echterhoff S, Norra C et al (2012) Loudness dependence of auditory evoked potentials (LDAEP) in clinical monitoring of suicidal patients with major depression: a pilot study. Eur Arch Psychiatry Clin Neurosci. doi:10.1007/s004 06-012-0297-8

10. Sawa M, Yamashita H, Fujimaki K, Okada G, Takahashi T et al (2012) Depressive symptoms and apathy are associated with psychomotor slowness and frontal activation. Eur Arch Psychiatry Clin Neurosci. doi:10.1007/s00406-012-0296-9

11. Fischer R, Kasper S, Pjrek E, Winkler D (2012) On the application of light therapy in German-speaking countries. Eur Arch Psychiatry Clin Neurosci. doi:10.1007/s00406-011-0286-3

12. Retz W, González-Trejo E, Römer KD, Philipp-Wiegmann F, Reinert $\mathrm{P}$ et al (2012) Assessment of post-excitatory long-interval cortical inhibition in adult attention-deficit/hyperactivity disorder. Eur Arch Psychiatry Clin Neurosci. doi:10.1007/s00406-0120299-6

13. Eich D, Angst J, Frei A, Ajdacic-Gross V, Rössler W et al (2012) A new rating scale for adult ADHD based on the Symptom Checklist 90 (SCL-90-R). Eur Arch Psychiatry Clin Neurosci. doi:10.1007/s00406-011-0288-1

14. Guo L-H, Westerteicher C, Wang X-H, Kratzer M, Tsolakidou A et al (2012) SORL1 genetic variants and cerebrospinal fluid biomarkers of Alzheimer's disease. Eur Arch Psychiatry Clin Neurosci. doi:10.1007/s00406-012-0295-x

15. Linden M, Muschalla B (2012) Standardized diagnostic interviews, criteria, and algorithms for mental disorders: garbage in, garbage out. Eur Arch Psychiatry Clin Neurosci. doi:10.1007/ s00406-012-0293-Z 